\title{
Active RFID and ESP8266 based Child Monitoring System
}

\author{
Chirag Yadav \\ Department of Computer \\ Engineering \\ MITCOE, Pune
}

\author{
Ankit Pandey \\ Department of Computer \\ Engineering \\ MITCOE, Pune
}

\author{
Mayank Saraogi \\ Department of Computer \\ Engineering \\ MITCOE, Pune
}

\author{
Shubham Tribhuwan \\ Department of Computer \\ Engineering \\ MITCOE, Pune
}

\begin{abstract}
One of the most prevalent problems in hospitals of India is the infants being abducted or getting mismatched from their respective parents. Thus a smart infant monitoring system based on RFID is proposed in this paper to reduce the potential risks. The smart infant monitoring system can be used in neonatal department in hospitals with integrated RFID modules. The smart infant monitoring system is composed of:
1. Child monitoring system (CMS)
2. Server Room System (SRS)

\section{Keywords}

Passive RFID reader ; Active RFID tag ; Server room ; CMS (child monitoring system); ESP8266 Wi-Fi module; Android; Arduino; Bluetooth module;

\section{INTRODUCTION}

One of the biggest problem nowadays in hospital is the new born babies getting abducted and infants getting mismatched due to lack of ability to distinguish between features of an individual infant. In order to prevent such situation in hospitals, RFID based infant monitoring system can help. By organizing and synchronizing technologies like Radio frequency Identification and Wi-Fi modules(ESP 8266, android Arduino etc); by using these technologies we provide a system which allows the new born babies being monitored every time without interrupting the regular care of the infants.

In this paper, discussion is based upon the architecture of the system. It covers the details of granularity of the system and then their integration and finally the project working.

\section{LITERATURE SURVEY}

In the current scenario, monitoring of the new born infants is done manually. The details of the infant are recorded manually on papers and verification is done by manually checking the records of the infants with their respective mothers. The security and safety of the infants is taken care of manually by the hospital staff by keeping a timely check in the neonatal department. So to increase the safety and minimize the man power, we are developing a system in which infants will be monitored using the RFID based system.

\section{CHILD MONITORING SYSTEM (CMS)}

To avoid the abduction of new born babies or getting swapped, the main objective of child monitoring system is to effectively integrate a system that could monitor infants through information technology. By using Radio Frequency Identification (RFID) technology, android module, a Bluetooth module and an active RFID module embedded in such a way that a system can be generated that could ensure the complete safety of the infant.

The proposed Child Monitoring System will solve two basic problems:

- Swapping of the infants

- Abduction of infants from the hospitals

How it will ensure that the infants are in the room?

For this purpose, the CMS is equipped with customized active RFID tags. The tag will be continuously sending the data to the RFID reader. In case of maximum count getting reduced in the server, the entries will be updated in the centralized database and hence, we will notified if there is a variation in the total number of infants present in the neonatal department.

How this system will ensure that when the alarm should be raised?

When the maximum count of the infant entries present in the centralized database gets reduced, the system will indicate the particular UID which is not being sensed by the reader and hence alarm will be raised.

The architecture comprises of

1) RFID based Infant Room Healthcare System (RIHS)

2) Server Room System (SRS)

\subsection{RFID Based Infant Room Healthcare System (RIHS)}

This system is installed in the neonatal rooms of the hospital. It comprises of three different modules which together senses the unique identification number (UID) from the tags attached to an infant and sends the data to the server room system. Following are the modules that RIHS comprises of:

1) Android Module (AM)

2) Arduino-Bluetooth Reader Module (ABRM)

3) ESP8266 Module (ESPM)

\subsubsection{Android Module (AM)}

This is a single module placed in each infant room in the hospital. Its main function is to obtain the unique identification number (UID) from Arduino-Bluetooth Reader Module and connect to the IP address of the Server Room System. 


\subsubsection{Arduino-Bluetooth-Reader Module (ABRM)}

This module comprises of an Arduino microprocessor which is attached with a Bluetooth module and an RFID reader. This module takes UID from RFID reader and sends it to the Arduino Module through Bluetooth .The reader used here is a passive RFID reader.

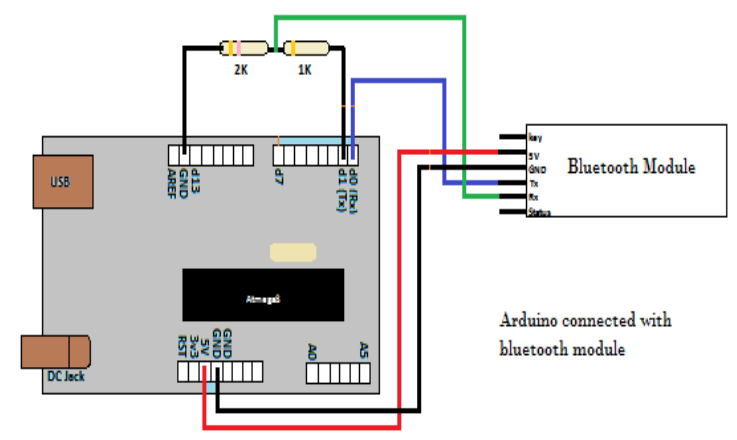

Fig 1: ABRM diagram

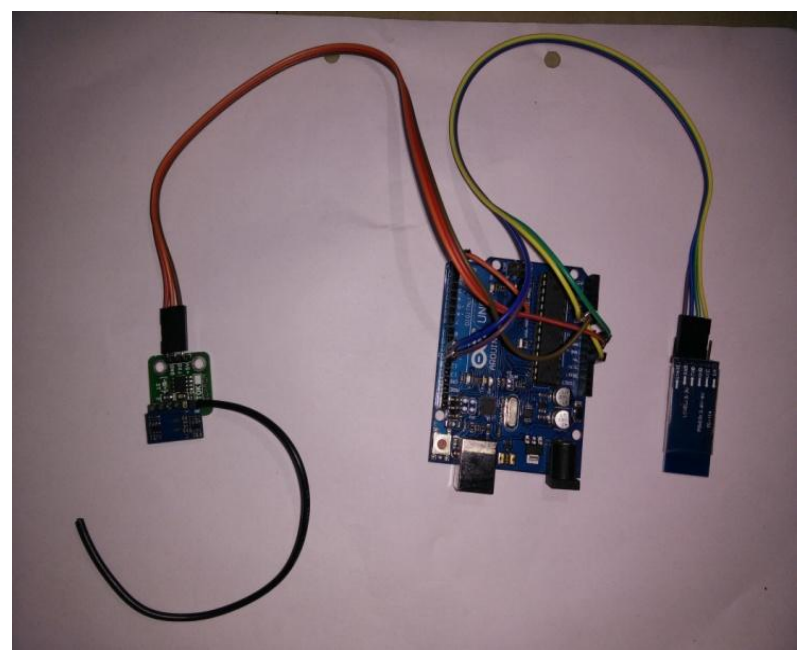

3.1.3 Customized RFID Tag (CRT)

This module comprises of an ESP $8266 \mathrm{Wi}-\mathrm{Fi}$ module .This module is attached in such a way with an active RFID tag such that it if someone tries to tamper with the tag, alarming signal will be send to the Android Module (AM).

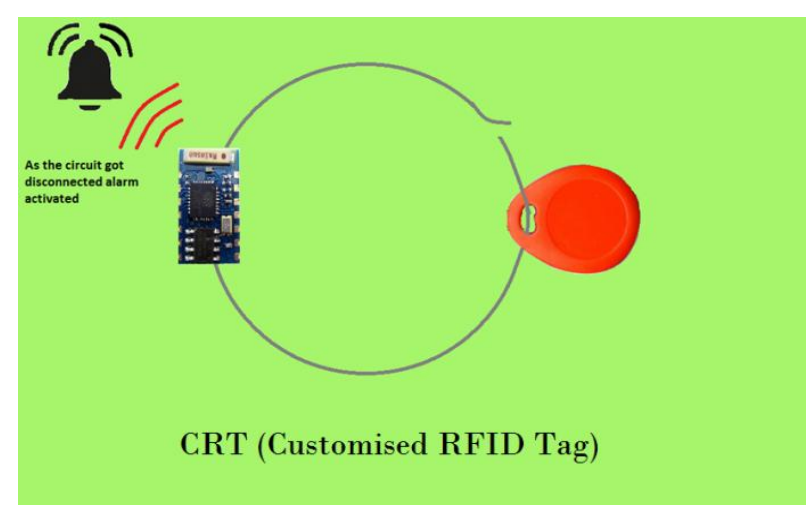

Fig 2: CRT diagram

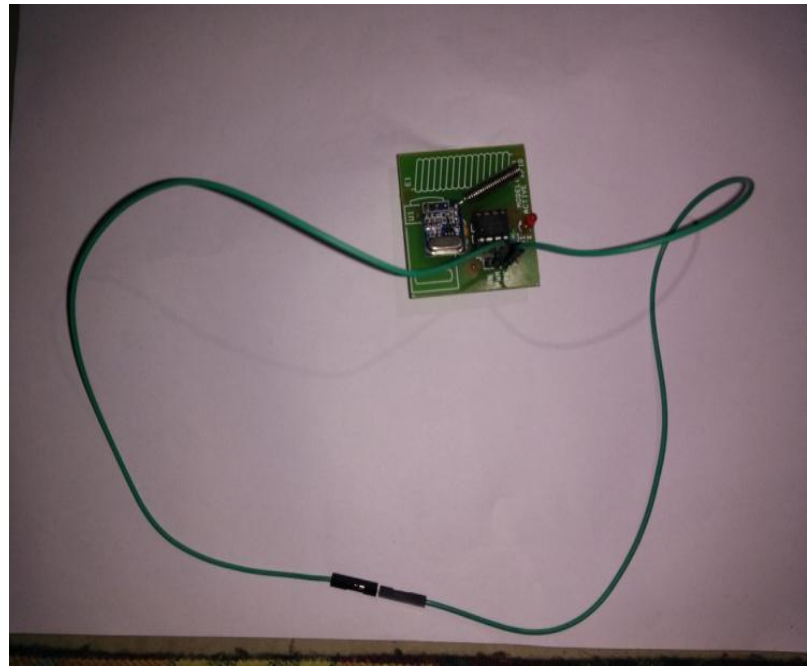

\subsection{Server Room System (SRS)}

This system is installed in the server room. The main function of this system is to monitor the data coming from RFID based Infant Room Healthcare System (RIHS).It comprises of the following modules:
1) Alarm System Module (ASM)
2) Data Entry Module (DEM)
3) Apache Tomcat Servlet Module (ATSD)

\subsubsection{Alarm System Module (ASM)}

This system comprises of an alarm buzzer. It will trigger the alarm if any case of abduction or tag tampering occurs. It takes data from the Apache Tomcat Servlet Module (ATSM).

\subsubsection{Data Entry Module (DEM)}

This module is prepared using NetBeans to take the details of the infants such as Unique Identification number (UID), Blood group, mother's name, father's name, birth mark etc.

\subsubsection{Apache Tomcat Servlet Module (ATSM)}

This module helps in creating a server system which obtains data continuously from the Android Module (AM) and makes entry in the database. This module also checks the database entries in order to decide whether to raise an alarm or not. 


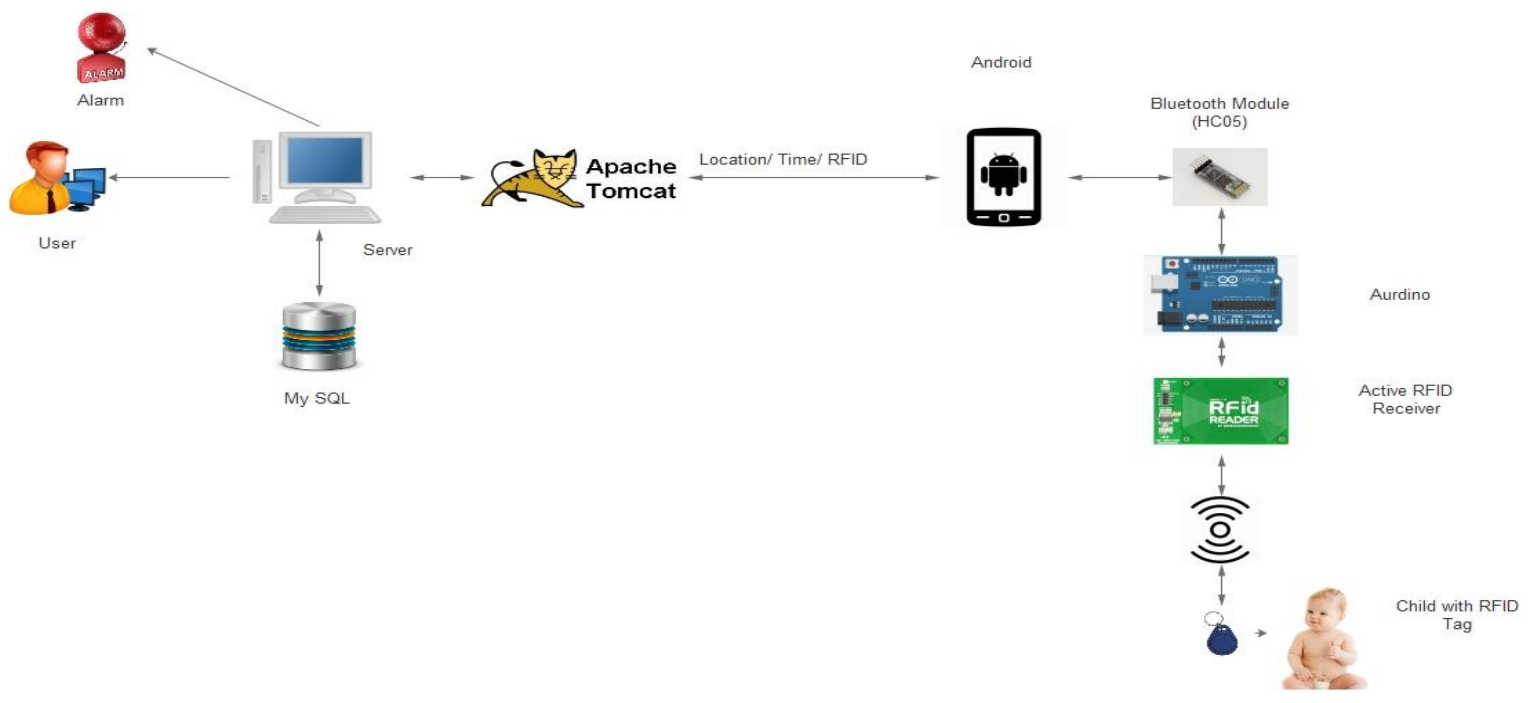

Fig 3: Architecture diagram

\section{RESULT AND DISCUSSIONS}

Cushioned and waterproof active RFID wristband will be assigned to the new born babies at the time of initial registration .All the necessary details of infant will be stored in the wristband (e.g. Mother name, father name, blood group, date of birth, time of birth, birth mark etc.) The details can be used to match the babies with their respective mothers. The positions of infants are continuously monitored by the RFID reader. The data can be read through RFID reader without interrupting the baby care.

\begin{tabular}{|c|c|c|c|}
\hline S.no & $\begin{array}{c}\text { Cut the RFID } \\
\text { Tag }\end{array}$ & $\begin{array}{c}\text { Alarm } \\
\text { Raised }\end{array}$ & Observation \\
\hline 1 & Yes & Yes & Rescued \\
\hline 2 & Yes & Yes & Rescued \\
\hline 3 & Yes & Yes & Rescued \\
\hline 4 & Yes & Yes & Rescued \\
\hline 5 & Yes & Yes & Rescued \\
\hline S.no & $\begin{array}{c}\text { Move the Tag } \\
\text { out of the room }\end{array}$ & $\begin{array}{c}\text { Alarm } \\
\text { Raised }\end{array}$ & Observation \\
\hline 1 & Yes & Yes & Rescued \\
\hline 2 & Yes & Yes & Rescued \\
\hline 3 & Yes & Yes & Rescued \\
\hline
\end{tabular}

Accuracy $=100 \%$

\section{CONCLUSION AND FUTURE SCOPE}

After testing of the smart monitoring system, following conclusions can be stated:

1) The smart infant monitoring system can detect any unauthorized handling of the infant and keep a check on every infant in the hospital.
2) We can prevent the mismatching of the infants by authenticating them with the details stored in the tag at the time of registration.

3) It is a straight forward way of securing the infants.

For future scope, security of the system can be extended by adding biometric module for the hospital staff so that only the authorized person can only enter the room. Further automation in doors for enhanced security can be programmed.

\section{REFERENCES}

[1] Shou-Hsiung Cheng, Department of information management, Chienko Technology University, Taiwan. An intelligent infant healthcare system of vital signs integrated by active RFID. In proceedings of the 2013 International Conference on Machine Learning and Cybernetics, Tianjin,2013

[2] Faisal Hussain, Shakeel Durrani, Hassan Mahmood, Horizon technologies, Islamabad, Pakistan. RFID \& WSN based integrated maternity ward monitoring system. 978-1-4799-3043-2/13/\$31.00/2013 IEEE

[3] Shou-Hsiung Cheng, Chun-Jung Lin, Department of information management, Chienko Technology University, Taiwan. A real time location and infant monitoring system based on active RFID. In Proceedings of the 2012 International Conference on Machine Learning and Cybernetics, Xian, 2012.

[4] Jui-Chen Huang, Department of Health Business Administration, Hungkuang University, Taichung city, Taiwan. . In Proceedings of the 2012 International Conference on Machine Learning and Cybernetics, Xian.

[5] Chun-Jung Lin, Department of Computer Science and Information management, Hungkuang University, Taichung city, Taiwan. . In Proceedings of the 2012 International Conference on Machine Learning and Cybernetics, Xian, july,2012.

[6] Imran Jattala, Nassar Ikram, Horizon technologies, Islamabad, Pakistan. RFID \& WSN based integrated maternity ward monitoring system. 978-1-4799-30432/13/\$31.00/2013 IEEE 
[7] "Kidnapping: Newborn kidnapped from hospital, "The Express Tribune, para 1,july 2013,[online] Available:http/:tribune.com.pk/story/575384/kidnapping -newborn-kidnapped-from-hospital/[ Accessed July 14,2013]

[8] A. Mahadevan and J. barker, “Embracing Radio Frequency Identification (RFID): Introduction and Analysis Using the Five Pillar Foundation Framework" ,2005 Asian Conference on sensors and the international Conference on new techniques in Pharmaceutical and Biomedical research,pp26-30,5-7 sep.2005.

[9] Wills S. and Helal S., "RFID Information Grid and Wearable Computing Solution to the Problem of Wayfinding for the Build User in a Campus Environment". Proceeding of the ninth annual IEEE International Symposium on Wearable Computers, Osaka, Japan, October 2005.

[10] R. Manoharan, G. Balamurugan and B rajmohan, "Embedded automated crash reporting system in vehicles based on SMS \&MMS with fish CAM eye camera," International Conference on Radar communication and Computing (IRCC),2012,pp.307-11,21-22Dec. 2012.

[11] P. Dickinson, K. Appiah, A. hunter and S. Ormston, "An FPGA based infant monitoring system", In proc. Of IEEE International Conference on Field-Programmable Technology,pp 315-316,Dec 11-14,2005
[12] Tzeng S, Chen W, Pai F. "Evaluating the business value of RFID: evidence from five case studies", International Journal 601-13. Apr 2008

[13] Kumiko 0., Sakiko 0., Lucila, O. M., Jiroshi T., "Smart medical environment at the point of care: Auto-tracking clinical interventions at the bed side using RFID technology", Computers in Biology and Medicine, Vol. 40, 20 10. p545-554

[14] Kim M. and Chong, N. Y., "Enhancing RFID Location Sensing using a Dual Directional Antenna", 6 th Asian Control Conference. Bali, Indonesia, pp.18-20, July, 2006

[15] Miller, L.E., Wilson, P.F., Bryner, N. P. , Francis, M. H. , Guerrieri, 1. R. , Stroup, D. W. and Berndt K. L., "RFID-Assisted Indoor Localization and Communication for First Responders", Proceeding of the International Symposium on Advanced Radio Technologies, March, 2006

[16] Lionel M.Ni, Yunhao L., Yiu C. L. and Abhishek P. P., "LANDMARK: Indoor Location Sensing Using Active RFID". Wireless Networks 10, 70 1-7 10, 2004

[17] Lee HJ, Lee, MC. "Localization of mobile robot based on radio frequency identification devices". In: SICE-ICASE, international joint conference, p. 5934-9. October 2006. 